\title{
Research in brief: Empagliflozin for patients with heart failure and preserved ejection fraction
}

\author{
Authors: Rajan S Pooni ${ }^{A}$ and Tevfik F Ismail ${ }^{B}$
}

KEYWORDS: empagliflozin, heart failure, preserved ejection fraction

DOI: 10.7861/clinmed.rib.22.1.1

\section{Background}

Summary of Anker SD, Butler ], Filippatos G et al. Empagliflozin in heart failure with a preserved ejection fraction. N Engl J Med 2021;385:1451-61.

Heart failure with preserved ejection fraction (HFpEF) is a term used to describe patients with clinical 'symptoms and signs of heart failure with [a left ventricular ejection fraction (LVEF)] $\geq 50 \%$ ' and is thought to encompass about $50 \%$ of all heart failure patients. ${ }^{2}$ In common with heart failure with reduced ejection fraction ( $\mathrm{HFrEF}$ ), it is associated with significant morbidity and mortality, but in contrast, no treatment has been shown to improve prognosis for HFpEF., 2,3

Recent randomised controlled trials have provided an evidence base for sodium-dependent glucose cotransporter-2 (SLGT2) inhibitors as prognostic medications for patients in HFrEF. Previously thought to reduce heart failure-related hospitalisation and adverse renal events in patients solely with type 2 diabetes, further trials have shown SGLT2 inhibitors to improve outcomes in heart failure regardless of diabetes status. ${ }^{4-6}$ The mechanism by which SGLT2 inhibitors provide cardiorenal benefit remains unclear. The anti-hyperglycaemic actions of SGLT2 inhibitors cannot fully explain their mechanism given that other antidiabetic medications have greater hypoglycaemic effects without an impact on heart failure prognosis. Proposed mechanisms of actions include reduction in sodium and water retention in the proximal renal tubule leading to improving renal function, which in turn may reduce afferent sympathetic supply to the heart and reduce cardiac inflammation.?

While the benefits of SGLT2 inhibitors in HFrEF are now established and in nascent treatment guidelines, their role in managing patients with HFpEF is unclear. ${ }^{2}$ In this summary, we

Authors: Ainternal medicine trainee, St Bartholomew's Hospital,

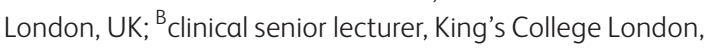
London, UK and consultant cardiologist, Guy's and St Thomas' NHS Foundation Trust, London, UK review a recent multicentre randomised controlled trial (EMPERORPreserved) that evaluated the benefits of the SGLT2 inhibitor empagliflozin in patients with apparent HFpEF.

\section{Study review}

After screening 11,500 patients across 622 centres, a total of 5,988 patients underwent randomisation, with 2,997 patients assigned to the intervention group (empagliflozin $10 \mathrm{mg}$ daily) and 2,991 to the placebo group. Key inclusion criteria included patients with a New York Heart Association (NYHA) class of II-IV, a preserved (or mildly reduced) ejection fraction (ie, $\mathrm{EF}>40 \%$ ) and an N-terminal - pro hormone B-type natriuretic peptide (NT-proBNP) level of $>300 \mathrm{pg} / \mathrm{mL}$ (without atrial fibrillation (AF)) or $>900 \mathrm{pg} / \mathrm{mL}$ (with AF). Patients with acutely decompensated heart failure, recent myocardial infarction or significant renal impairment were excluded. Baseline characteristics, including the number of patients with diabetes ( $\sim 49 \%$ of patients enrolled), were similar across both groups.

The primary endpoint was a composite of cardiovascular death or heart failure-related hospitalisation. The median duration of followup across both groups was 26.2 months. The primary endpoint was observed in $13.8 \%$ in the intervention group compared with $17.1 \%$ in the placebo group (intention-to-treat analysis).

A secondary outcome evaluating the decline in estimated glomerular filtration rate (eGFR) was also noted to be slower in the intervention group compared with the placebo group $\left(-1.25 \mathrm{~mL} / \mathrm{min} / 1.73 \mathrm{~m}^{2}\right.$ per year versus $-2.62 \mathrm{~mL} / \mathrm{min} / 1.73 \mathrm{~m}^{2}$ per year). Such results are similar to findings in previous studies involving patients with HFrEF (LVEF $<40 \%$ ), albeit the observed renal benefits were significantly lower in this study. With uncertainty existing for the relationship between change in the eGFR slope and major adverse renal outcomes, the authors performed a prospective pooled analysis of patients with LVEF $<40 \%$ and LVEF $\geq 40 \%$, concluding that ejection fraction influences the effects of empagliflozin on major renal outcomes. ${ }^{8,9}$ Specifically, while the decline in eGFR was noted to be slower in both groups, this only correlated with lower serious renal outcomes (ie, profound and sustained decreases in eGFR or renal replacement therapy) in the LVEF $<40 \%$ population. As such, using eGFR slope analysis may have limitations as a surrogate for drug-induced renal outcomes. With a recent metaanalysis suggesting that eGFR slope is a strong predictor of longterm renal outcome in early chronic kidney disease (CKD), further study is needed in this area. ${ }^{10}$ 
While touted as an HFpEF trial, it is interesting that this trial enrolled patients with both mildly reduced ejection fraction (EF $40 \%-50 \%)$ and preserved ejection fraction $(>50 \%)$. This is relevant as it is likely that patients with mildly reduced ejection fraction behave more like patients with HFrEF than true HFpEF. ${ }^{11}$ The use of a LVEF of $40 \%$ as a dividing line is artificial and reflects the ability of LVEF values below this value to identify patients in historical observational studies at increased risk of mortality with heart failure post-myocardial infarction. ${ }^{12}$ This, in turn, was used to identify cohorts of patients with asymptomatic left ventricular dysfunction with high event rates most likely to accrue benefit from an angiotensin-converting enzyme inhibitor in the original clinical trials for these agents, motivated by the need to keep sample sizes and trial costs feasible. ${ }^{13}$ Subsequent heart failure trials have used this cut-off or similar values for the past 30 years, creating the 'condition' HFrEF.

Pre-specified exploratory subgroup analysis showed that patients with $\mathrm{EF} \geq 60 \%$ at baseline derived no statistically significant benefit (hazard ratio (HR) 0.87; but with $95 \%$ confidence interval (CI) $0.69-1.10$ ) with respect to the primary outcome. Similarly, the benefit was of borderline significance in the $50 \%-60 \%$ range (HR $0.80 ; 95 \%$ CI $0.64-0.99)$. This is of relevance given likely differences in $\mathrm{EF}$ reference ranges for men and women and the imprecision with which LVEF is measured when evaluated by echocardiography.

Despite a statistically significant primary outcome, on further evaluation, cardiovascular mortality was not significantly lower in the empagliflozin group. Furthermore, a similar finding was observed in the trial involving empagliflozin in patients with HFrEF (EMPERORReduced). Statistically significant benefit appeared to be driven by reduced heart failure-related hospitalisation rather than mortality.

\section{Key points}

> As for patients with HFrEF, SGLT2 inhibitors appear to improve some cardiorenal outcomes in heart failure patients with LVEF $\geq 40 \%$.

$>$ However, there was no difference in cardiovascular mortality between the treatment and placebo arms. Differences in outcomes appeared to be driven by reductions in hospitalisation for heart failure. The number of patients needed to treat (NNT) with empagliflozin to prevent one adverse cardiovascular outcome was $\sim 31$ (absolute risk reduction 3.3\%).

> Whether benefits accrue equally to patients with true HFpEF (defined as $L V E F \geq 50 \%$ ) or those with mildly reduced $E F$
$(40 \%-50 \%)$ remains unclear. Specific further prospective evaluation of patients with higher ejection fractions (ie, true $\mathrm{HFpEF}$ ), as opposed to mildly reduced ejection fractions, is needed.

> The decline in eGFR was slower in the treatment arm, however, it is unclear whether this is a relevant surrogate renal outcome.

\section{References}

1 Anker SD, Butler J, Filippatos $\mathrm{G}$ et al. Empagliflozin in heart failure with a preserved ejection fraction. N Engl J Med 2021;385:145161.

2 McDonagh TA, Metra M, Adamo M et al. 2021 ESC guidelines for the diagnosis and treatment of acute and chronic heart failure. Eur Heart J 2021:42:3599-726.

3 Solomon SD, McMurray JJV, Anand IS et al. Angiotensin-neprilysin inhibition in heart failure with preserved ejection fraction. N Engl J Med 2019;381:1609-20.

4 Zelniker TA, Wiviott SD, Raz I et al. SGLT2 inhibitors for primary and secondary prevention of cardiovascular and renal outcomes in type 2 diabetes: a systematic review and meta-analysis of cardiovascular outcome trials. Lancet 2019:393:31-9.

5 Packer M, Anker SD, Butler ] et al. Cardiovascular and renal outcomes with empagliflozin in heart failure. $N$ Engl J Med 2020;383:1413-24.

6 McMurray JJV, Solomon SD, Inzucchi SE et al. Dapagliflozin in patients with heart failure and reduced ejection fraction. $N$ Engl J Med 2019;381:1995-2008.

7 Lopaschuk GD, Verma S. Mechanisms of cardiovascular benefits of sodium glucose co-transporter 2 (SGLT2) inhibitors: A state-of-theart review. JACC Basic Transl Sci 2020;5:632-44.

8 Packer M. Pitfalls in using estimated glomerular filtration rate slope as a surrogate for the effect of drugs on the risk of serious adverse renal outcomes in clinical trials of patients with heart failure. Circ Heart Fail 2021;14:e008537.

9 Packer M, Butler J, Zannad F et al. Empagliflozin and major renal outcomes in heart failure. N Engl J Med 2021;385:1531-3.

10 Inker LA, Heerspink HJL, Tighiouart $\mathrm{H}$ et al. GFR slope as a surrogate end point for kidney disease progression in clinical trials: A metaanalysis of treatment effects of randomized controlled trials. J Am Soc Nephrol 2019;30:1735-45.

11 Butler ], Anker SD, Packer M. Redefining heart failure with a reduced ejection fraction. JAMA 2019;322:1761-2.

12 Multicenter Postinfarction Research Group. Risk stratification and survival after myocardial infarction. N Engl J Med 1983;309:331-6.

13 Pfeffer MA, Braunwald E, Moyé LA et al. Effect of captopril on mortality and morbidity in patients with left ventricular dysfunction after myocardial infarction. Results of the survival and ventricular enlargement trial. N Engl J Med 1992;327:669-77. 